\title{
Three steps approach for preoperative evaluation of tetralogy of Fallot patients: role of $128 \mathrm{MDCT}$
}

\author{
Sherif Abd El Fattah Moustafa', Manar Mansour Hussein ${ }^{1 *} \mathbb{D}$, Amina Ahmed Sultan', Maha Mohamed Zaki Bilal', \\ Mohamed Adel Fetoh El Gamal ${ }^{2}$ and Donia Mohamed Sobh
}

\begin{abstract}
Background: Tetralogy of Fallot is the most common congenital cyanotic heart disease. Advances in surgical repair recently facilitate survival of the affected patients into adulthood with good quality of life. Despite imaging plays crucial role in diagnosis of TOF patients, no single diagnostic modality suffices for complete evaluation of TOF. Thus, different diagnostic tools should be used alone or in combination according to patient's clinical question. This study aims to find out the agreement between the echocardiography and MDCT in evaluation of TOF patients prior to surgical repair.

Results: Three-step approach used in scanning TOF patients. Firstly, classic TOF findings, MDCT was comparable to echocardiography in overriding aorta $(k=0.6), \mathrm{VSD}(k=0.8), \mathrm{RVH}(k=0.4)$, and sub valvular pulmonary stenosis $(k=$ 0.6). Echocardiography is superior to MDCT in evaluation of valvular stenosis $(k=0.4)$. MDCT was superior to echocardiography in supravalvular pulmonary stenosis. Secondary, surgical relevant findings, McGoon ratio calculated in 94.2\%, small and major APCs in $45.5 \%$ and $28.5 \%$ respectively, PDA in 35\% and anomalous coronary artery in 5.1\%. Thirdly, other TOF-associated anomalies, common: ASD (34.3\%) and RAA (28.3\%), and persistent LSVC (10.3\%). Uncommon: situs ambiguous (2.5\%), PAPVR (1.2\%), and IVC anomalies (3.8\%). MDCT was superior to echocardiography in surgical relevant findings and other TOF-associated anomalies.
\end{abstract}

Conclusion: MDCT is a crucial imaging tool for extra cardiac evaluation of TOF patients prior to surgical repair.

Keywords: Tetralogy of Fallot, Multidetector computer tomography, Congenital cyanotic heart disease, Right ventricular outflow tract

\section{Background}

Tetralogy of Fallot is the most common congenital cyanotic heart disease, it accounts for $7 \%$ to $10 \%$ of congenital defects, affecting males and females equally and occurring in 3 to 5 of every 10,000 live births [1]. It is essentially created by anterior and superior deviation of the conal septum and manifested in a group of anatomical malformations with four characteristic features consisting of: interventricular communication, also known

\footnotetext{
* Correspondence: manarmansour2011@yahoo.com

'Diagnostic and Interventional Radiology, Mansoura University, Mansoura, Egypt

Full list of author information is available at the end of the article
}

as a ventricular septal defect (VSD), biventricular aortic root connection that overrides the muscular ventricular septum, obstruction of the right ventricular outflow tract (RVOT), and right ventricular hypertrophy (RVH) [2].

TOF has a broad anatomical spectrum, the obstruction can be located at the right ventricular infundibulum, in severe forms the pulmonary valve is atretic, the pulmonary arteries are absent and the lung is supplied by aortapulmonary collaterals [3].

Surgical management differs from conventional surgery in the milder conditions to more complex procedures in the severe forms with reconstruction of the pulmonary arteries. Therefore, the level and degree of 
obstruction of the right ventricular outflow in addition to the degree of pulmonary arteries development are outstanding prerequisites to surgical correction [3].

Although echocardiography and catheter-directed cardiac angiography are generally accepted as the primary imaging techniques for $\mathrm{CHD}$, they have many limitations that preclude accurate evaluation prior to surgical correction [4]. Small acoustic window of the echocardiography precludes the evaluation of certain anatomical structures (e.g., ascending aorta, right ventricle (RV) size and function, aberrant coronary anatomy, pulmonary arteries, and major aortopulmonary collateral arteries) [5]. High cost, ionizing radiation burden, invasive nature, and prolonged sedation anesthesia are the main disadvantages of cardiac catheterization [2].

CT and MRI are important emergent complementary diagnostic tools [4]. Both of them can be used as problemsolving tools, especially in the evaluation of preoperative cardiovascular anatomy [6]. However, CMRI is expensive, long scan time requiring lengthy sedation and anesthesia, and the post-processing is time-consuming. In addition to artifacts from non-MR-compatible implants, and poor evaluation of lungs and airways [7].

Very short acquisition times rendering superior spatial and temporal resolution, and diminished motion artifacts are provided by recent generations MDCT. MDCT allows depiction of the cardiac, extra-cardiac abnormalities including the lungs and airways. It allows for excellent 3D description of cardiovascular anatomic structures [8].

Preoperative computed tomography imaging in tetralogy of Fallot patients is directed at confirmation of the diagnosis and differentiation of TOF from other common mixing disorders such as a common arterial trunk, transposition of the great vessels with a ventricular septal defect, or a double-outlet right ventricle (DORV) [3].

Further goals of preoperative imaging are to establish the severity of the primary anatomical lesion, the associated anomalies, and the degree of functional disturbance that in turn determine appropriate timing of surgical intervention. The surgical approach itself may be altered by anatomical relationships of cardiac, coronary, and extra-cardiac structures, such as a right-sided aortic arch, MAPCAs, and variant coronary anatomy [2].

The role of our study is to highlight the role of MDCT compared to echocardiography in evaluation of TOF patients prior to surgical repair, regarding the intra-cardiac and extra-cardiac anomalies through an organized radiological approach.

\section{Methods}

\section{Patients}

This prospective study included 77 consecutive pediatric patients that were diagnosed as tetralogy of Fallot by echocardiography and referred by their pediatric cardiologists and cardiothoracic surgeons. They were referred to the CT unit at the Radiology Department, in our institution to undergo MDCT using Philips CT with 128 detector rows (Philips, Ingenuity Core, Philips Healthcare, and the Netherlands). This was carried out at the period from April 2016 till October 2018.

This study was approved by the international review board (IRB) and written consents were obtained from all patients' parents for sedation and contrast material administration. Patients with impaired renal function and hypersensitivity to contrast material were excluded.

\section{ECG-gated multi-detector cardiac CT angiography}

ECG-gated technique was used, in this pediatric age group, the heart rate $70-130 \mathrm{bpm}$. Sedation was given to younger patients (below 5 years, or who appear noncooperative) using the choral hydrate with a dose of 50$100 \mathrm{mg} / \mathrm{kg}$ to avoid motion-induced artifacts. Non-ionic iodinated contrast agent (Iohexol $350 \mathrm{mg} / \mathrm{ml}$, omnipaque, GE Healthcare, Ireland) was injected at a dose of $0.5-2 \mathrm{~mL} / \mathrm{kg}$ body, at a rate of $1-2 \mathrm{~mL} / \mathrm{s}$ via 20 -22-gage catheter into peripheral vein. To reduce artifacts from undiluted contrast material and consequently the total amount of contrast material, a saline bolus chasing technique was applied in most of the cases.

We used the bolus tracking technique to determine the imaging delay in ECG-gated CTA. A region of interest was placed in the left ventricle, and a threshold attenuation of $200 \mathrm{HU}$ is set. Data acquisition was performed in a cranio-caudal direction from the level of the thoracic inlet down to the upper abdomen. The data acquisition was performed with continuous imaging table motion and spiral acquisition. For gapless data acquisition, low pitch with slice collimation $128-0.625 \mathrm{~mm}$, gantry rotation time $0.4 \mathrm{~s}$ was used.

Increased heart rate in pediatric population precludes the prospective ECG-gated technique in order to obtain high-resolution studies for smaller structures evaluation as coronaries. Thus, the radiation dose was kept as minimum as possible by reducing the tube current potential and adjust it to patient's age. Tube voltage was $80-100$ KvP, and tube current was 150-200 mA.

Examination of the heart was then performed and sequential series of images (0-90\% of R-R interval), in arterial and subsequent phase of enhancement taken to ensure opacification of both sides of the heart and all extra-cardiac vessels.

\section{Image processing}

The source images were analyzed on separate workstations (Intellispace portal, version 5, Philips Healthcare). The 2D Multi-planar reformations (MPR) and volume rendering (VR) images were reconstructed to aid in the diagnosis. 


\section{Image analysis}

The images were interpreted by two radiologists simultaneously (3 and 10 years' experience in cardiac MDCT imaging). The sequential segmental approach of Van Praagh 1972 was used in the analysis of the images. It includes situs determination, cardiac looping, relation of the great vessels, and atrioventricular and ventriculoarterial concordance. Degree of aortic overriding was visually assessed (Fig. 1). Origin of the pulmonary artery and conal anatomy were reviewed. Cardiac chambers were evaluated regarding dilatation or hypertrophy. The presence of VSD, its type, and size. The thoracic aorta was evaluated regarding direction of the aortic arch (either left sided or right sided) and detection of the branching pattern. The thoracic aorta was measured at different levels: at the aortic root, ascending aorta, aortic arch, and aorta at diaphragm. In the presence of patent ductus arteriosus (PDA) (Fig. 2), it was assessed regarding origin, morphology, length, and termination. Its diameter was measured at the aortic and pulmonary ends. Evaluation of the MAPCAs was done with documentation of their origin, course, termination, and caliber (Figs. 3 and 4). The pulmonary arteries were evaluated regarding (Fig. 5) origin and branching pattern, 5 measurements of pulmonary were taken annulus, proximal and distal MPA, both right and left PAs prior to first-order branches. McGoon ratio was calculated, $X=(L P A+R P A) / D A o$. Where LPA = left pulmonary artery, $\mathrm{RPA}=$ right pulmonary artery, and Dao $=$ descending aorta at the level of diaphragmatic crus.
The radiological findings were classified into (a) Classic TOF findings, (b) surgical relevant findings, and, (c) other associated congenital anomalies. Overriding aorta, VSD, RV hypertrophy, and RV outlet obstruction (RVOTO) are the classic findings (Fig. 1). The surgical relevant findings that would impact surgical decision include McGoon ratio calculation, presence or absence of the aorto-pulmonary collaterals, presence or absence of the PDA, and presence or absence of the coronary artery anomalies (Fig. 6). The other associated congenital anomalies as situs ambiguous (Fig. 5), aortic arch variants and anomalies, anomalous pulmonary venous connection, and systemic venous anomalies (Fig. 6).

\section{Statistical analysis and data interpretation}

Statistical analysis was performed using SPSS version 22 (SPSS Inc., Chicago, IL). Data was tested for normality using Kolmogorov-Smirnov test. Continuous data were presented as mean \pm standard deviation (SD). Qualitative data were described using number and percent. Quantitative data were described using the median (minimum and maximum). $P$ values $<0.05$ were considered significant. Agreement between the CT and echo findings was performed by weighted-kappa measurements. Agreement levels were interpreted as $0.00-0.20$ negligible, 0.21-0.40 fair, $0.41-0.60$ moderate, $0.61-0.80$ good, and 0.81-1.00 almost perfect agreement.

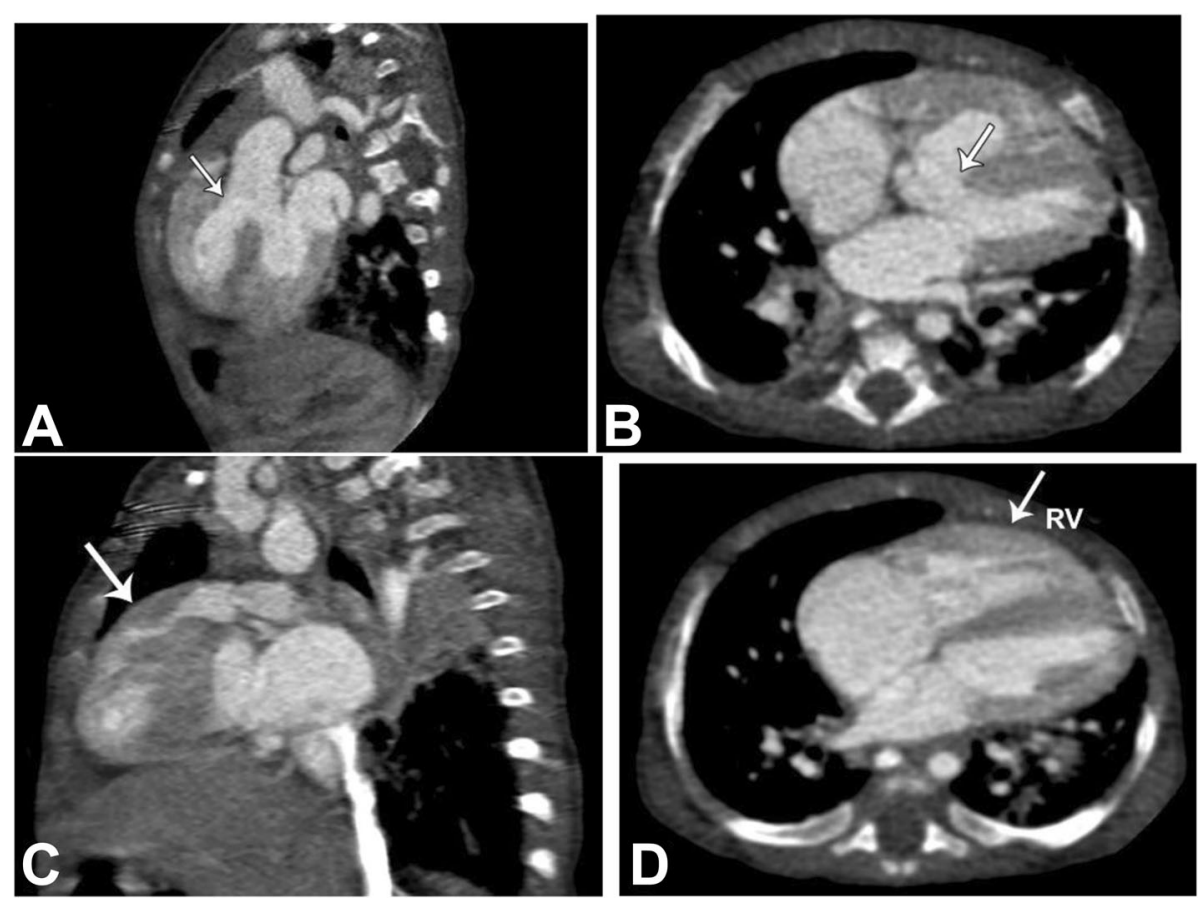

Fig. 1 Two-year-TOF boy underwent MDCT shows: a Coronal oblique MPR image showing aorta overrides both RV: LV = 50: 50\% (arrow). b Axial oblique MPR image is showing sub aortic outlet VSD (arrow). c Sagittal oblique MPR image is showing hypertrophied infundibulum with RVOT narrowing (arrow). d Axial image showing dilated hypertrophied RV (arrow) and dilated right atrium 

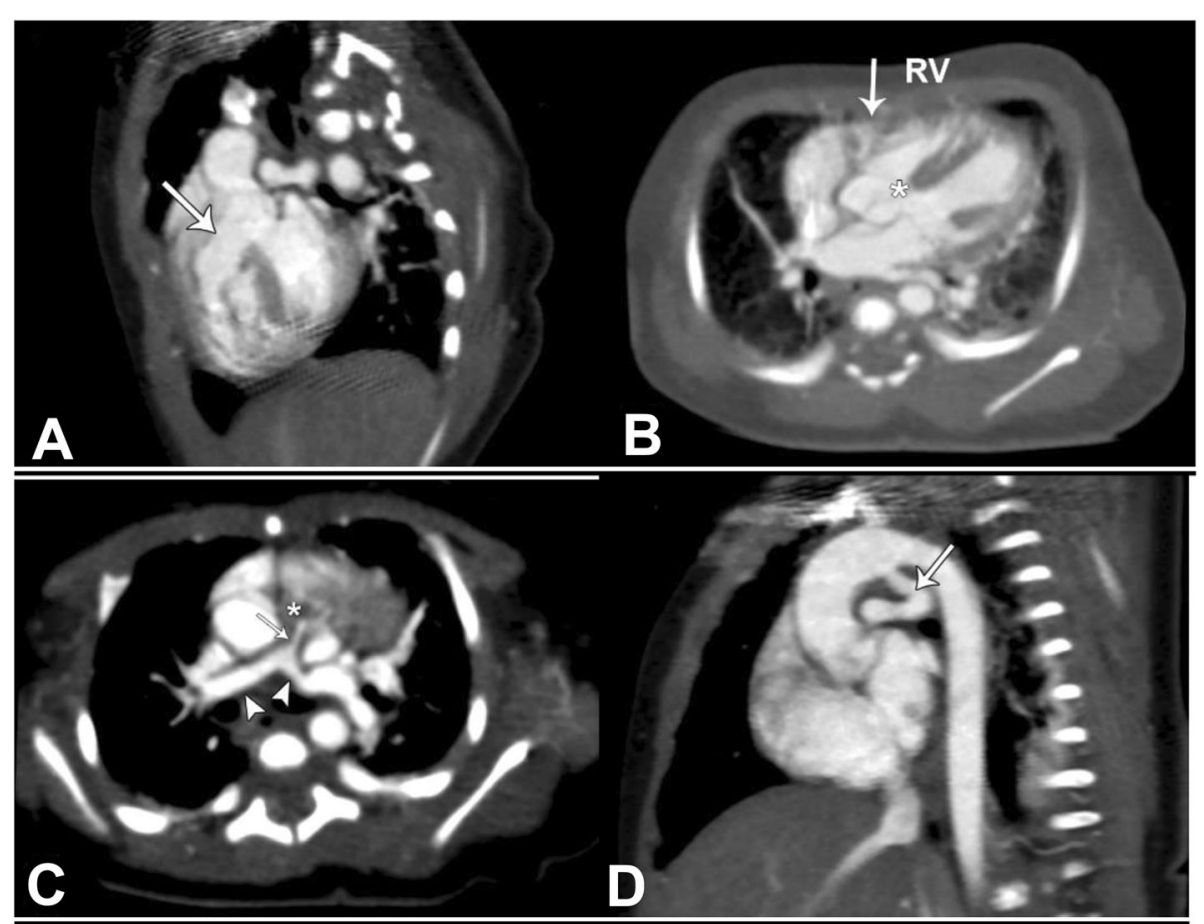

Fig. 2 Two-month-TOF boy. a Aorta overrides both ventricles (arrow). b Subaortic outlet VSD (asterisk) and hypertrophied RV. c Atresia of the pulmonary valve (asterisk) and severely hypoplastic MPA (arrow), small-sized right and left pulmonary arteries (arrowheads). d U-shaped PDA (arrow) connecting the distal aortic arch to the left PA

\section{Results}

This prospective study included 77 pediatric patients $(40$ males and 37 females), who were diagnosed as tetralogy of Fallot based on clinical and echocardiographic findings. Their ages ranged between 12 days and 16 years.

\section{Agreement between echocardiography and $\mathrm{CT}$ in assessment of the RVOTO \\ Assessment of level of RVOTO}

As shown in Table 1 RVOTO can be classified into subvalvular, valvular, and supra-valvular levels of obstruction. The supra valvular level can be divided into either central MPA or its branches (right and left pulmonary arteries). There was Good agreement $(k=0.65, p<0.03)$ between echocardiography and MDCT in subvalvular pulmonary stenosis, fair agreement $(k=0.4, p<0.13)$ in valvular stenosis and left pulmonary artery assessment. No agreement was found between echo and CT regarding focal stenosis MPA and RT PA, as they could not be detected by echocardiography.

Combined subvalvular, valvular, and supravalvular pulmonary stenosis was the most common type of RVOTO in 36 patients (46.7\%), followed by combined subvalvular and valvular level in $41.5 \%$. While $3.8 \%$ showed isolated subvalvular, $2.59 \%$ had isolated valvular, and $2.59 \%$ had isolated supravalvular obstruction.

\section{Agreement between echocardiography and CT in assessment of pulmonary artery size}

There was a strong positive correlation as regards MPA measurement by Echo and MDCT $(r=0.857$ and $p<0.000)$. While there was a moderate positive correlation between the RT PA measurement by echo and CT $(r=0.609$ and $p<0.000)$. Weak positive correlation was found in the left PA measurement $(r=$ 0.401 and $p<0.000$ ) between echocardiography and CT.

\section{Agreement between echocardiography and CT in assessment of overriding aorta}

There was good agreement ( $p=0.029$ and $k=0.6$ ) between the Echo and MDCT in the evaluation of aortic overriding. Degree of aortic overriding of the interventricular septum was reported to be $(\mathrm{RV} / \mathrm{LV})=50 / 50 \%$ in 66 cases $(85.7 \%)$ by echo versus 60 cases $(77.9 \%)$ by MDCT. While (RV/LV) $=60 / 40$ in 11 cases (14.28\%) by echo versus $15(19.5 \%)$ by MDCT. There was one case $(1.3 \%)$ that showed $\mathrm{RV} / \mathrm{LV}=40 / 60 \%$ aortic overriding by echo versus 2 cases (2.6\%) by MDCT.

\section{Agreement between echocardiography and CT in assessment of ventricular septal defect}

All cases showed subaortic outlet VSD, there was very good agreement ( $p=0.02$ and $k=0.87$ ) between the MDCT and Echo as regards the evaluation of the number of VSDs. Seventy-two cases (93.5\%) had single VSD 

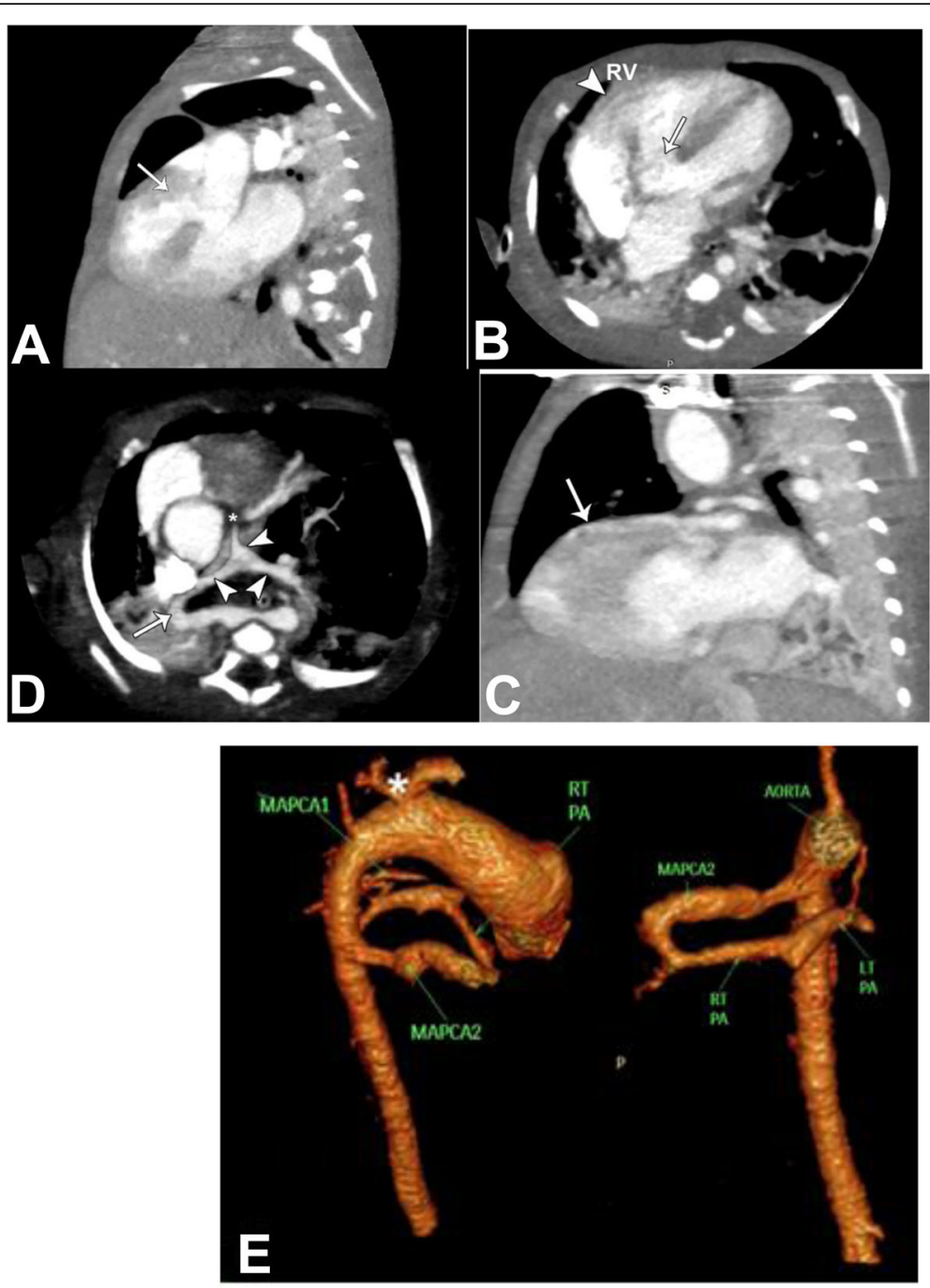

Fig. 3 Three-month-TOF boy. a Coronal MPR image of overriding aorta (arrow). b Axial image at the level of subaortic outlet VSD (arrow) with mildly hypertrophied RV (arrowhead). c Sagittal MPR image showing marked narrowing of RVOT (arrow). d Axial MIP image revealed atresia of the pulmonary valve (asterisk), marked hypoplasia of MPA and its two branches (arrowhead). MAPCA (arrow) is seen arising from the anterolateral aspect of the descending thoracic aorta connected to the end of the right PA. $\mathbf{e}$ VR images of the thoracic aorta showing the aortopulmonary collaterals (MAPCA 1 and MAPCA2), bovine AA arch is noted (asterisk)

by Echo versus 73cases (94.8\%) by MDCT. Five cases had more than one VSD by echo versus 4 cases by MDCT.

\section{Agreement between echocardiography and CT in} assessment of the right ventricular hypertrophy All cases had RVH, 25 cases (32.5\%) had mild right ventricular hypertrophy $(\mathrm{RVH})$ and 52 cases $(67.5 \%)$ had marked RVH by CT with fair agreement $(k=0.41)$ between echo and MDCT.

\section{Agreement between echocardiography and CT in} assessment of the McGoon ratio

As shown in Table 2, there was poor agreement between Echo and MDCT in McGoon ratio calculation $(k=0.3$ and $p<0.001$ ). McGoon ratio (mean \pm SD) $1.29 \pm 0.9$ by echo versus $1.8 \pm 0.81$ with poor correlation in between $(r=0.23$ and $p<0.04)$.

\section{Agreement between echocardiography and $\mathrm{CT}$ in} assessment of major aortopulmonary collateral arteries (MAPCAs)

MDCT was superior to echo in detection of the MAPC As. MAPCs were identified in 22 cases by CT compared to 8 cases by echo. Only delicate collaterals were identified in 35 cases by CT. The total number of MAPCAs was 57 collaterals with average caliber (mean $\pm \mathrm{SD})=$ $3.65 \pm 1.44$. 

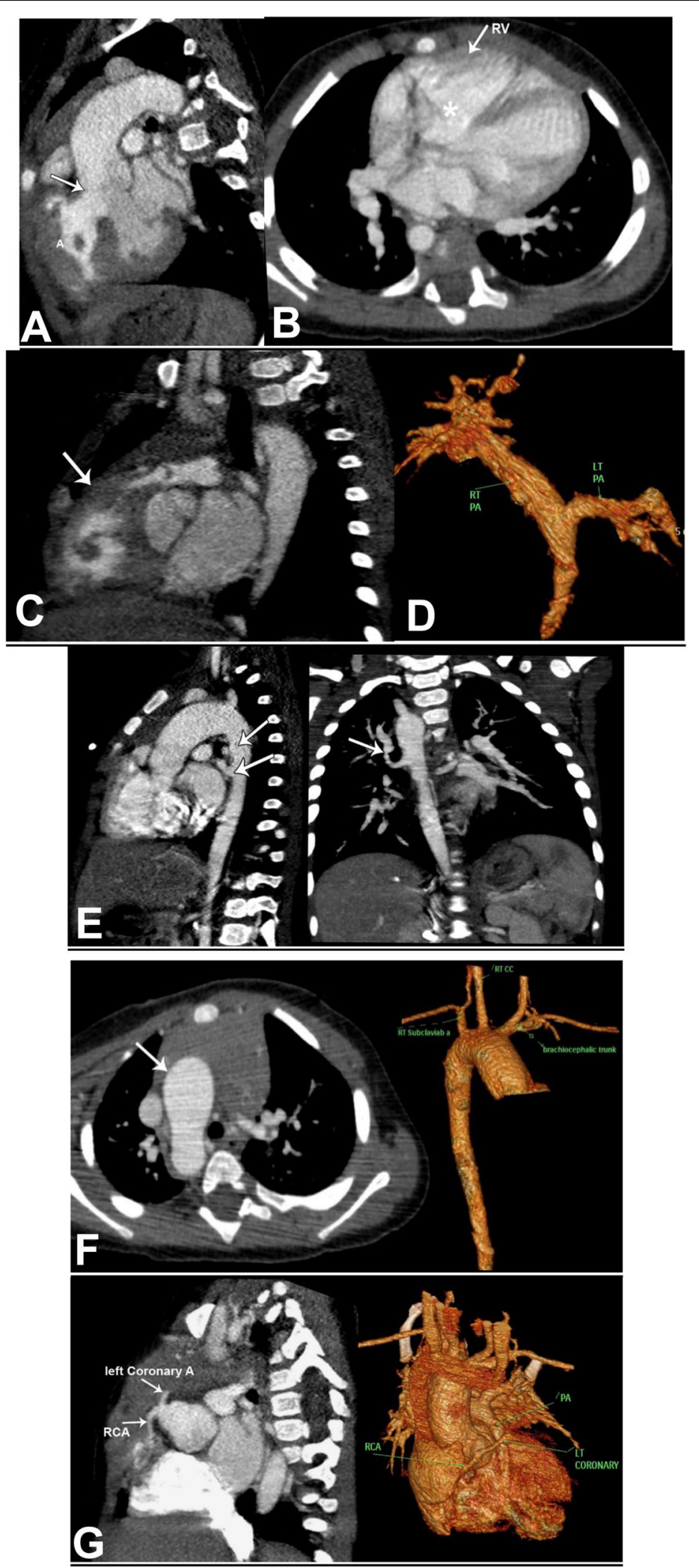

Fig. 4 (See legend on next page.) 
(See figure on previous page.)

Fig. 4 Ten-month-TOF boy. a Overriding aorta (arrow). b Subaortic outlet VSD (asterisk) and hypertrophied RV. c Infundibular muscle

hypertrophy with marked RVOT narrowing (arrow). Thickened pulmonary valve appears in the image (asterisk). d Normal MPA and its two

branches arrows in VR image. e Artopulmonary collaterals arising from the descending aorta and coursing to both hila (arrows). f Right-sided AA

with mirror image branching pattern. $\mathbf{g}$ Anomalous origin of coronary arteries, both RCA and LCA arise by common ostium (right coronary

ostium). Left coronary artery passes in front of the RVOT

\section{Agreement between echocardiography and CT in assessment of PDA}

There was statistically significant strong agreement $(p=$ $0.001, k=0.8$ ) between Echo and MDCT in the detection of PDA. Twenty cases showed PDA by echo versus 27 cases by CT. There was statistically significant moderate correlation $(p=0.01, r=0.6)$ in measurement of the PDA diameter between CT and echo (average PDA diameter $=0.98 \mathrm{~mm}+/-1.4$ by Echo and $1.19 \mathrm{~mm}+/-1.9$ by MDCT).

\section{MDCT in assessment of coronary arteries}

There were 4 (5.1\%) cases with anomalous coronary arteries origin, one showed common origin of all coronaries from single ostium from the right coronary sinus, then divides, and LAD passes across the RVOT. The second case showed RCA and the left main coronary artery arise from the right coronary sinus by two separate origins. The LAD also passes across the RVOT. The third case showed hypertrophied conal branch from the RCA passes across the RVOT. The last case showed high take off with angled origin of the RCA.

\section{MDCT in assessment of associated anomalies}

Regarding other associated anomalies, there were two cases that had situs ambiguous was identified in 2 cases and 28 cases $(36.4 \%)$ had ASD.

Table 3 shows the types of aortic arch as well as the variations in branching pattern. There were 55 cases with left-sided aortic arch (AA) versus 22 cases had right-sided AA. The most common branching pattern in left-sided AA was the normal branching pattern followed by bovine AA in 31 (40\%) and 16 (20.8\%) cases respectively. While the most common branching pattern in right-sided AA is mirror image branching pattern in 12 (15.5\%) cases.

Partial anomalous pulmonary venous return was identified in one case $(1.2 \%)$ in which the right upper pulmonary vein drained directly into SVC. Three (3.8\%) cases showed absent IVC with azygos continuation and confluence of the hepatic veins into a single trunk connected to the right atrium. Persistent (left superior vena cava) LSVC was found in 8 cases (10.3\%). Left-sided SVC was found in one case (situs ambiguous-left sidedness) and retro aortic brachiocephalic vein was found in 3 cases (3.8\%).

\section{Discussion}

Tetralogy of Fallot (TOF) represents $7-10 \%$ of congenital heart disease (CHD), 30-50\% of congenital cyanotic heart disease. Advances in surgical repair recently facilitate survival of the affected patients into adulthood with good quality of life [9].

Organized imaging approach is needed not only for identification of the four major malformation of TOF but importantly for precise information about associated extra-cardiac vascular anatomical abnormalities. That information is crucial for surgeons in the formulation of surgical strategies to improve surgical planning and outcomes [10].

No single diagnostic modality suffices for complete evaluation of TOF at any stage. Different diagnostic tools should be used alone or in combination according to the clinical question, the age, size, and clinical condition of the patient, the availability, cost radiation burden of each modality, and the need for sedation and the contraindications for each patient [9].

Cardiovascular magnetic resonance imaging (CMR) is an outstanding imaging tool in assessment of TOF patients, it provides detailed assessment of cardiac and extracardiac morphology, functional analysis (RV size and function, pulmonary valve competence, and pulmonary arterial flow) without radiation or iodinated contrast agents, and is suitable for numerous longitudinal followups. However, it is expensive, long scan time requiring lengthy sedation and anesthesia, and the post-processing is time-consuming. Other disadvantages include artifacts from non-MR-compatible implants, poor evaluation of lungs and airways, and the risk of nephrogenic systemic sclerosis with gadolinium-based products [7].

In the current era, MDCT offers excellent spatial and reasonable temporal resolution providing static and cine imaging as well as $3 \mathrm{D}$ reconstructions, thus, it will be very helpful and reliable tool in TOF evaluation [9].

In our study and as regards the role of MDCT in TOF prior to surgical repair, three-step approach was followed, including step (1) recognition of the classic TOF findings, step (2) searching for the surgically relevant findings, and step (3) scanning for other associated congenital anomalies.

\section{Role of MDCT in recognition of classic TOF findings}

The degree of overriding aorta was assessed in all patients. This finding is crucial not only in diagnosis of TOF but also for surgical planning of VSD patch closure as patients with double outlet RV will need much larger patch to baffle the blood from the LV to the aortic root 

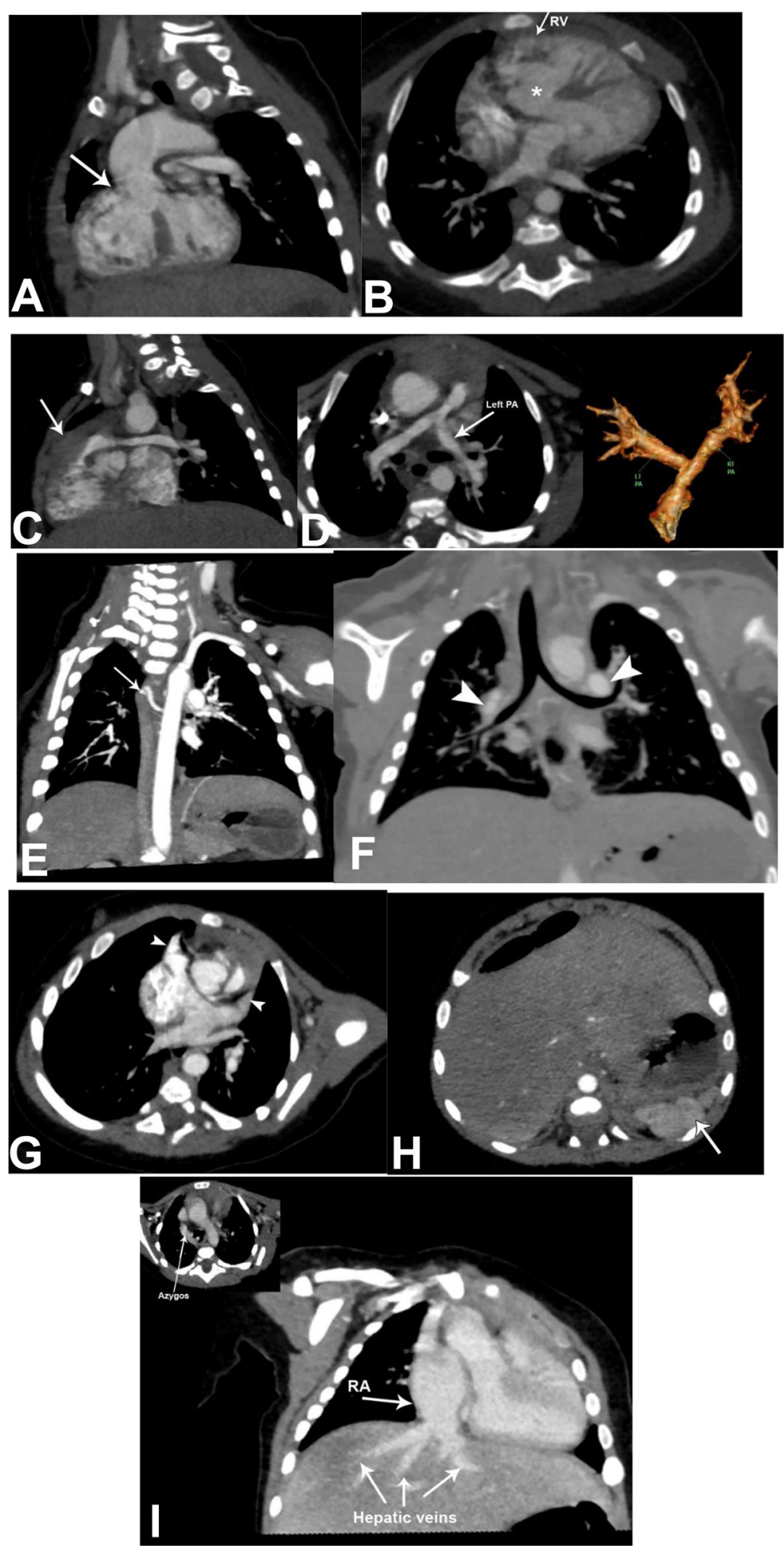

Fig. 5 Two-month-TOF girl with left isomerism. a Overriding aorta (arrow). b Subaortic outlet VSD (asterisk), loss of the aorta-mitral continuity is noted (curved arrow) and mildly dilated RV. c Hypertrophied infundibular muscle with RVOT stenosis (arrow). d Small-sized pulmonary arteries. e MAPCA arising from the descending thoracic aorta and supplying right lung hilum (arrow). f Coronal oblique MPR minMIP image showing left bronchial morphology (hyparterial) of both bronchi. $\mathbf{g}$ Both atria of left atrial morphology (arrowheads). h Mid-line liver, lobulated spleen with small splenules, and left sided stomach. i Interrupted IVC with azygos continuation, hepatic veins converge to drain directly into the right atrium 

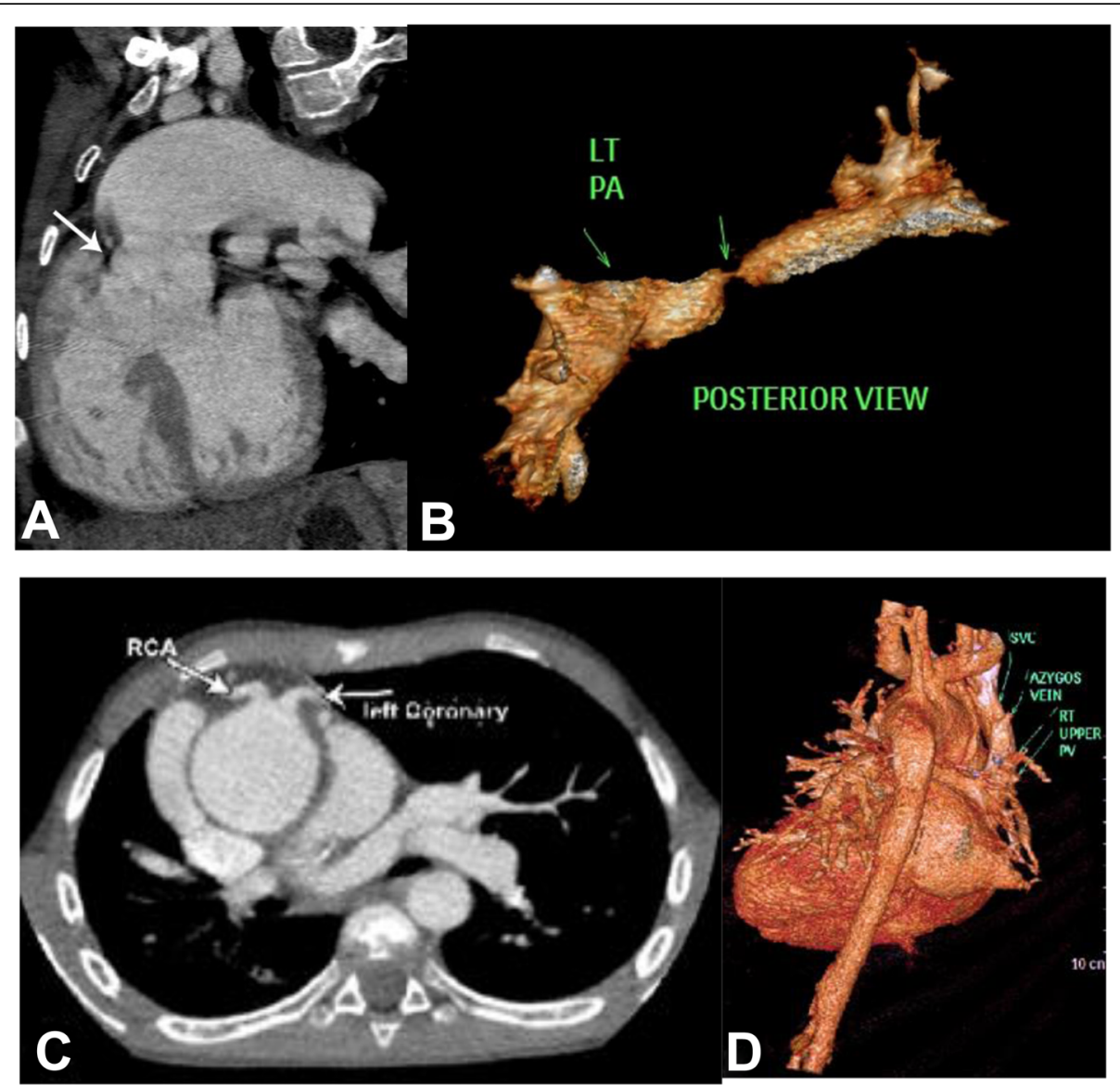

Fig. 6 Nine-year-old boy had history of TOF-pulmonary atresia and MBT shunt. a Aorta overriding both ventricles with dilated aortic root and ascending aorta (asterisk). b Pulmonary atresia (absent MPA) with confluent pulmonary arteries. Severe narrowing of the pulmonary confluence (arrow). c RCA and left main coronary artery arise by two separate adjacent ostia from the right coronary sinus. d Posterior view of the heart, right upper pulmonary vein drained directly at the SVC inferior to entry of the azygos vein (PAPVR)

[11]. In 60 patients (78\%), the degree of the overriding was nearly equal (50:50\%). While in 15 patients (19.5\%), the aorta arised more from the RV mimicking double outlet RV (TOF variant). Only in 2 patients $(2.6 \%)$, the aorta arised more from the LV. Statistically significant moderate agreement $(k=0.6)$ was found between the MDCT and echocardiographic findings; however, the variability in between could be attributed to the visual observation method used in both modalities which would differ depending on the angle that the observer views the aortic valve in relation to the crest of the ventricular septum as explained by Barron et al. [11].

The interventricular septal defects were assessed in all patients. Subaortic outlet perimembranous VSDs were found in all cases show with statistically significant strong agreement with echocardiography. However, additional muscular VSD was detected in 4 cases by MDCT opposite 5 cases by echocardiography. This would be attributed to the very small intermuscular defect that was easier to be detected by color Doppler rather than
MDCT. This relatively came in agreement with Barron et al. [11].

The right ventricular outflow obstruction (RVOTO), severity, and level of RVOT obstruction should be adequately evaluated to determine the surgical management of TOF and the age at which it will be carried out [12]. In our study, we found three levels for RVOT obstruction, sub-valvular (infundibular), valvular, and supra valvular (congenital pulmonary stenosis). The subvalvular stenosis was the most commonly affected level in 71 cases $(92.2 \%)$. This came in agreement with Agrawal et al. [13] who studied the sites of the pulmonary stenosis at TOF and concluded the sub-valvular pulmonary stenosis was commonly affected at $96 \%$, followed by valvular stenosis at $82 \%$, and lastly supra valvular stenosis at $33.2 \%$.

The patients usually had more than one level of obstruction. Studying level of obstruction in every case showed that combined level of obstruction is much more common than isolated level of obstruction. 
Table 1 Agreement between echo and CT in evaluation of the RVOTO

\begin{tabular}{|c|c|c|c|c|c|c|}
\hline \multirow{2}{*}{$\begin{array}{l}\text { RVOTO } \\
\text { Number }\end{array}$} & \multicolumn{2}{|l|}{$\mathrm{ECHO}$} & \multicolumn{2}{|l|}{ CT } & \multirow{2}{*}{$\begin{array}{l}\text { Kappa } \\
\text { agreement }\end{array}$} & \multirow{2}{*}{$\begin{array}{l}p \\
\text { value }\end{array}$} \\
\hline & $N=77$ & $\%$ & $N=77$ & $\%$ & & \\
\hline Sub valvular & $\# N=71$ & & $\# N=71$ & & 0.65 & $0.03^{*}$ \\
\hline - Antero septal deviation without hypertrophy & 5 & 6.6 & 4 & 5.3 & & \\
\hline - Mild infundibular hypertrophy & 40 & 53.3 & 43 & 57.3 & & \\
\hline - Marked infundibular hypertrophy & 26 & 34.6 & 24 & 32 & & \\
\hline Valvular & ${ }^{* *} N=64$ & & ${ }^{* *} N=64$ & & 0.4 & $0.013^{*}$ \\
\hline - Thickened PV & 53 & 82.8 & 57 & 89 & & \\
\hline - Atresia of PV & 11 & 17.2 & 7 & 11 & & \\
\hline \multicolumn{7}{|l|}{ Supra-valvular } \\
\hline$M P A$ & ${ }^{* * *} N=36$ & & ${ }^{* * *} N=30$ & & & \\
\hline - Hypoplastic artery & 21 & 85.3 & 23 & 73.3 & & \\
\hline - Focal stenosis & 0 & 0 & 4 & 13.3 & & \\
\hline - Absent & 15 & 41.7 & 4 & 13.3 & & \\
\hline$R T P A$ & ${ }^{\#} N=21$ & & ${ }^{\#} N=24$ & & & \\
\hline - Hypoplastic artery & 18 & 85.7 & 18 & 75 & & \\
\hline - Focal stenosis & 0 & 0 & 3 & 12.5 & & \\
\hline - Absent & 3 & 14.3 & 3 & 12.5 & & \\
\hline Left PA & $\# N=25$ & & $\# N=30$ & & 0.456 & $0.023^{*}$ \\
\hline - Hypoplastic artery & 22 & 84.6 & 20 & 64.6 & & \\
\hline - Focal stenosis & 2 & 7.7 & 8 & 25.8 & & \\
\hline - Absent & 1 & 3.8 & 2 & 6.6 & & \\
\hline
\end{tabular}

Combined sub-valvular, valvular, and supra valvular obstruction was found in 36 cases (46.7\%), and combined sub-valvular and valvular obstruction in 32 cases (41.5\%), isolated sub-valvular obstruction was only found in 3 cases, and isolated valvular obstruction in 2 cases.

The supra valvular obstruction including the MPA and its branches revealed complex anomalies. Four cases had pulmonary atresia (5.1\%), one case had atresia of MPA only, two cases showed atresia of the MPA, and its branches, and only one case showed absent MPA and right PA. While the remaining patients showed different degrees of pulmonary artery stenosis: the most frequent anomaly is combined MPA, RT, and left PA arteries stenosis at 14 cases (18\%) followed by isolated left PA stenosis at seven cases (9\%). This relatively came in

Table 2 Agreement between echo and $C T$ in McGoon ratio calculation

\begin{tabular}{|c|c|c|c|c|c|c|}
\hline \multirow{3}{*}{$\begin{array}{l}\text { MCGON ratio } \\
\text { Number }\end{array}$} & \multirow{2}{*}{\multicolumn{2}{|c|}{$\begin{array}{l}\text { Echo } \\
N=77\end{array}$}} & \multirow{2}{*}{\multicolumn{2}{|c|}{$\frac{C T}{N=77}$}} & \multirow[t]{3}{*}{ Agreement } & \multirow[t]{3}{*}{$p$ value } \\
\hline & & & & & & \\
\hline & $N$ & $\%$ & $N$ & $\%$ & & \\
\hline Not calculated & 21 & 27.3 & 4 & 5.2 & \multirow[t]{2}{*}{$K=0.3$} & \multirow[t]{2}{*}{$<0.001^{*}$} \\
\hline Calculated & 56 & 72.7 & 73 & 94.2 & & \\
\hline Mean \pm SD & \multicolumn{2}{|c|}{$1.29 \pm 0.9$} & \multicolumn{2}{|c|}{$1.84 \pm 0.81$} & $r=0.23$ & $P=0.04^{*}$ \\
\hline
\end{tabular}

agreement with Zakaria et al. [14] who reported incidence of combined MPA stenosis and its branches $17 \%$ as the most common pulmonary artery anomaly. While it disagrees with Sheikh et al. [15] who stated that isolated left pulmonary stenosis was the most common abnormality in $10 \%$. This may be attributed to larger number of patients (about 5000) included in the study who underwent cardiac catheterization.

There was difficulty in assessing the degree of agreement between echocardiography and MDCT in evaluation of MPA and RT PA. MDCT could detect focal stenosis at 7 cases (MPA 4 cases and RT PA 3 cases), which was missed by echocardiography. Poor agreement between both modalities regarding left PA evaluation was identified secondary to superiority of MDCT to echocardiography in pulmonary arteries evaluation. Hrusca et al. [12] reported CT accuracy in detection of pulmonary artery defects in $92.1 \%$ of cases. Hu et al. [10] reported accuracy of CT up to $100 \%$ in detection of supra valvular pulmonary stenosis, they compared their result to cardiac catheterization. Apostolopoulou et al. [9] recommended MDCT evaluation of complex pulmonary artery defects in TOF patients for better visualization pulmonary arteries anatomy, size, and arborization. 
Table 3 Types of aortic arch and variation in branching pattern in our study

\begin{tabular}{|c|c|c|c|}
\hline Aortic arch & Branching pattern & $N$ & $\%$ \\
\hline \multirow[t]{6}{*}{ Left-sided AA } & Normal branching pattern & 31 & 40.4 \\
\hline & Bovine arch & 16 & 20.8 \\
\hline & Aberrant RSCA & 2 & 2.6 \\
\hline & Direct origin of the vertebral artery & 3 & 3.8 \\
\hline & Bovine arch with aberrant RSCA & 3 & 3.8 \\
\hline & Total & 55 & 71.6 \\
\hline \multirow[t]{6}{*}{ Right-sided AA } & Mirror imaging branching pattern & 12 & 15.58 \\
\hline & Bovine arch & 3 & 3.8 \\
\hline & Common origin of both CCAs and separate origin of both SCAs & 3 & 3.8 \\
\hline & Aberrant LSCA & 3 & 3.8 \\
\hline & Aberrant LSCA, common origin of both CCAs \& direct origin of the vertebral artery. & 1 & 1.29 \\
\hline & Total & 22 & 28.32 \\
\hline Total & & 77 & $100 \%$ \\
\hline
\end{tabular}

As regards the right ventricular hypertrophy (RVH), we noted that all cases showed RVH by MDCT, 52 cases (67.5\%) show mild RVH, and 25 cases (32.5\%) show marked RVH. While by echocardiography, 58 patients were reported to have RVH by echocardiography with poor correlation in between, $k=0.46$. These findings agree with results of Ramanathan et al. [16] who stated that there was poor correlation between echocardiography and MDCT in evaluation of the right-sided heart either by quantitative or qualitative methods. On the other hand, quantitative measurements show very good correlation between both modalities regarding the left side of the heart. This was attributed to the limitation of the echocardiography in evaluation of the right ventricle, in addition to low sensitivity but high specificity of MDCT in evaluation of the cardiac chambers.

\section{Role of MDCT in assessment of the surgically relevant findings}

Besides to characterization of the pulmonary arteries, our study provided the surgeons important findings that could formulate the surgical decision, these findings were described as surgically relevant findings and include McGoon ratio calculation, presence or absence of the aortopulmonary collaterals, presence or absence of PDA, and presence or absence of coronary artery anomalies.

As regards McGoon ratio calculation, it is an applicable method to evaluate the pulmonary arterial size and pulmonary blood flow. When the McGoon ratio is adequate, it means enough pulmonary blood, and hence, total surgical correction is planned. Otherwise, palliative operation is suggested in case of inadequate McGoon ratio [17]. In our study, McGoon ratio was calculated in 73 cases by MDCT versus 56 cases by echocardiography with poor agreement in between. This came in agreement with Chen et al. [17] who stated that MDCT with reconstructed oblique images are reliable methods for McGoon ratio calculation when they compared it to the angiographic findings. While transthoracic echocardiography is limited in providing accurate measurements, secondary to small acoustic window, in consistent oblique angle at which it cuts the pulmonary arteries and posterior course of the left pulmonary.

Major aortopulmonary collateral arteries (MAPCAs) are usually present in the most severe forms of TOF. The surgical management of these collaterals aims to restore the normal pulmonary blood flow that is are readily variable depending on patient-specific anatomy [18]. We detected aortopulmonary collaterals at 57 cases(74\%). MAPCAs were seen at 22 cases (28.5\%) and delicate collaterals were seen at 35 cases (45.5\%). At a study done by Juneja et al. [19], they described incidence of the MAPCAs in TOF patients about (56\%), but that study included homogenous group of TOF patients with pulmonary atresia, while our study included various congenital pulmonary defects. All cases (22 cases) had origin from the descending thoracic aorta, while 7 cases $(31.8 \%)$ out of them show additional origin from the aortic arch vessels. All of them show mediastinal course without significant airway compromise and terminate into hila. These detailed quantitative and qualitative findings were not provided by echocardiography. Superiority of MDCT over echocardiography coincides with Chandrashekhar et al. [20] who stated that MDCT had the ability similar to catheterization in aortopulmonary collateral identification, while it is difficult to detect these collaterals by echocardiography mainly secondary to small field of view also. 
PDA is a common anomaly in TOF patients, especially in TOF with pulmonary atresia (TOF-PA), when there is ductal dependant pulmonary circulation. Stenting the PDA has gained acceptance for palliation in TOF-PA. However, the complex morphology of the PDA makes the stent implantation more challenging. Hence, this raises the role of imaging in better delineation of the morphology of the PDA [21]. MDCT could detect 27cases (35.1\%) with PDA opposite 25 cases (32.4\%) detected by echocardiography with strong agreement in between. Yet, their average caliber by CT $=1.19 \pm 1.9$ versus $0.98 \pm 1.4$ by echocardiography, with moderate agreement in between. This coincides with Shehata et al. [22] who stated the MDCT was superior to transthoracic echocardiography (TTE) in evaluation of PDA. Also, it was in accordance with Ishehara et al. [23] who recommended MDCT to evaluate doubtful cases for PDA with normal echocardiography, but this study was done in adult patients. While these findings differ from Leschka et al. [24] who reported that TTE is considered the method of choice for diagnosing PDA while MDCT has only a minor role.

Coronary artery anomalies are not uncommon in TOF and should be accurately assessed prior to surgical management. In ventriculotomy or trans annular repair when done to relieve the RVOT obstruction, the aberrant coronary artery passing across the RVOT is reliable to be injured accidentally with poor surgical outcomes [10]. We had 4 cases $(5.1 \%)$ with anomalous coronary arteries, one of them showed common origin of all coronaries from a single ostium that arise from the right coronary sinus, then divided into LAD passing across the RVOT and RCA in its normal course. It is worth noting that this anomaly had been reported by Beig et al. [25] as a very rare anomaly in the general population with incidence $(0.0240$ to $0.066 \%)$ with more frequent incidence in TOF patients (1.5 to 3.7\%).

The second case showed RCA and the left main coronary artery had arised from the right coronary sinus by two separate ostia. The LAD also passed across the RVOT. The third case showed hypertrophied conal branch from the RCA passing across the RVOT. While the last case revealed high take off with angled origin of the RCA. It is worth noting that these findings were not provided at all by echocardiography.

Incidence of coronary artery anomalies (5.1\%) coincides with literature as reported by Hrusca et al. [12] that varied from 5 to $12 \%$. However, in their study that had been done to detect anomalous coronary and pulmonary defects in TOF patients, they found coronary artery anomalies in 8 cases $(22.2 \%)$, they described two out of them with small conal branches from RCA. But these small conal branches are not crucial to be counted from the anomalies as they will not impact the surgery.
However, in a study by $\mathrm{Hu}$ et al. [10], they described the coronary artery anomalies at 2 cases $(2 \%)$ with sensitivity $100 \%$ and specificity $100 \%$ when compared to cardiac catheterization. Also in a study by Zakaria et al. [14], they described only one case of abnormal LAD origin. In our study, MDCT was superior to echocardiography in detection coronary artery anomalies, this coincides with Gotein et al. [26] and Shehata et al. [22].

\section{Role of MDCT in assessment of associated anomalies}

As regard TOF-associated findings, we found that MDCT is superior in delineation of the extracardiac vascular anomalies. There were 2 cases that showed abnormal situs (situs ambiguous) of abnormal viscero-atrial relationship. This finding was accidentally noted by MDCT and had not been detected in echocardiography. This may be explained that situs determination needs cardiac, bronchial and abdominal viscera evaluation which is more better by cross-sectional imaging. Although abnormal situs is a rare finding in TOF patients, it was also mentioned by Zakaria et al. [14].

In our study, there were 28 cases $(36.4 \%)$ that had interatrial septal defects (ostium secondum in 27 cases and ostium primum in only one case). The incidence of ASD in TOF patients is variable in literature, it was reported $3-5 \%$ by Beig et al. [25], $10 \%$ by Zakaria et al. [13], and $15 \%$ by Verma et al. [27]. It was difficult to assess the ASD at 9 cases by MDCT because of motion and respiratory artifacts while they were reported in echocardiography. This is coping with Yasunaga and Hamon [28] who stated that echocardiography is still the gold standard technique for evaluation of the interatrial septum, while MDCT could provide complementary role in large-sized ASD or in delineation of associated extracardiac anomalies. It was noted that $12 / 28$ cases (42.8\%) were associated with PDA. This incidence may raise concern to look for PDA in TOF with ASD.

The aortic arch variants and anomalies are not uncommon in the general population and much more common in patients of CHD. Despite AA variants are often asymptomatic and reported as an incidental finding, recently they have been found to pose a greater risk of hemorrhage and ischemia during surgery in the thorax [29]. Variations in the AA type and branching pattern are important to recognize before cardiothoracic surgeries or interventional procedures because they could affect the technique of some of them [30]. In our study, we have found many AA variants in TOF patients. Left-sided AA was found in 55 patients $(71.6 \%)$ and right-sided AA was found in 22 patients (28.3\%). Despite this incidence being cope with many review articles as Lapierre et al. [3] and Zakaria et al. [14] in which RAA incidence about 25 and 30\% respectively, this incidence is more or less in accordance with other authors as $\mathrm{Hu}$ 
et al. [10] and Tawfik et al. [30]. They reported incidence of RAA in TOF patients as 18.5 and $37 \%$ respectively.

The most common ramification pattern of the leftsided aortic arch was normal branching pattern (brachiocephalic trunk followed by left CCA and left SCA) followed by bovine arch (common origin of brachiocephalic and left CCA) by $40.4 \%$ and $22.8 \%$ respectively. This came in accordance with Tawfik et al. [30], their incidence was $36 \%$ and $16 \%$ respectively. In general, the most common ramification pattern of the RAA is aberrant left SCA. However, in our study, RAA with mirror imaging branching pattern was the most common ramification type of RAA by $54.5 \%$. This came in accordance with Hanneman et al. (2017) [31] who stated that RAA with mirror image branching is strongly associated with CHD in up to $98 \%$ of cases, including tetralogy of Fallot.

Anomalous pulmonary venous connection are rare but established association with TOF. Total anomalous pulmonary venous return (TAPVR) was reported in some case reports more frequent than partial anomalous pulmonary venous return (PAPVR). This is attributed to embryological basis explained by recent hypothesis. Awareness of anomalous pulmonary venous return in TOF patients is an important issue prior to surgical correction to preclude catastrophic outcomes [32]. PAPVR was detected in one case (1.2\%) which was not identified in echocardiography. In this case, two right superior and middle pulmonary veins were seen draining into the SVC, while the remaining right and left pulmonary veins drained normally into the left atrium. In a study done by Chan et al. [33], concluded that repaired TOF patients with unrepaired PAPVR were liable to more RV volume overload and more reduction in RV function.

Also, in our study, MDCT was superior to echocardiography in detection of the PAPVR, this came in accordance with Türkvatan et al. [34]. They stated that MDCT is more reliable than TTE in delineation of the PAPVR likely due to operator dependance and limited acoustic window in delineation of some anomalous pulmonary veins. This is more difficult when the anomalous veins are multiple branching, show high insertion to the SVC, or associated with complex congenital heart disease or enlarged left atrium.

Systemic venous anomalies were found uncommon in TOF patients in our study. It has been found that interrupted IVC with azygos continuation was incidentally noted in 3 cases (3.8\%). Two of them had associated with situs ambiguous. Although association between IVC anomalies and TOF had not been reported in literature, Zakaria et al. [14] had also reported one case with double IVC. Persistent LSVC was found in 8 cases (10.3\%), left-sided SVC with absent right SVC was found in single situs ambiguous (left isomerism) patient. The prevalence of PLSVC in TOF patients is uncommon, Ari et al. [35] reported in 5.9\%, and Nagasawa et al. [36] reported it in $5.1 \%$, these studies are not compatible to some extent with our results (10.3\%). Likely, it is attributed to using echocardiography in their studies. In our study, only five cases (4.9\%) were detected by echocardiography.

Although retroaortic brachiocephalic vein was described as a very rare anatomical variant in the general population $(2 / 100000)$ and $(5 / 1000)$ in CHD by Corno et al. [37], we have found 3 TOF patients (3.8\%) with retroaortic brachicephalic vein that also having rightsided AA. Lim et al. [38] published it as a case report in 3-month girl TOF patient using echocardiography. This may be attributed that it is easier to sequentially delineate this venous variant by MDCT than that by echocardiography. Awareness of this venous anomaly is beneficial to avoid transvenous pacemaker or central venous catheter placement from left-arm approach and to minimize perioperative risks [38].

\section{Limitations and recommendations}

Although CTA had advantages on morphological imaging, yet it is limited in volumetric and functional analysis of the cardiac chambers and pressure measurement. Taking into account these drawbacks, patients should be carefully selected for CT imaging, and strategies for radiation exposure reduction need to be applied.

\section{Conclusion}

MDCT plays crucial role in diagnosis and surgical planning of TOF patients. It provides superiority over echocardiography in detailed evaluation of the pulmonary arteries, aortopulmonary collaterals, PDA, anomalous coronary arteries, and common and uncommon extra cardiac vascular anomalies. The prescribed three-step approach helps the radiologists to provide the diagnostic data and not to miss the surgical relevant findings.

\section{Abbreviations}

TOF: Tetralogy of Fallot; VSD: Ventricular septal defect; RVOTO: Right ventricular outflow tract obstruction; MAPCAS: Major aortopulmonary collateral arteries; DORV: Double outlet right ventricle; TAPVR: Total anomalous pulmonary venous return; PAPVR: Partial anomalous pulmonary venous return

\section{Acknowledgements}

Not applicable.

\section{Authors' contributions}

AS and MM designed the research. MM performed the research; and wrote the manuscript. AS and ShA analyzed the collected data. AS, ShA, and DS revised data and manuscript. All authors read and approved the final manuscript.

\section{Funding}

Not applicable (no funding received for this study).

Availability of data and materials

Available on request with the corresponding author. 


\section{Ethics approval and consent to participate}

This study was approved by the research ethics committee of the Radiology Department of the Faculty of Medicine Mansoura University on 21 May 2016, Reference number of approval: MD.16.05.41

All patients included in this study gave a written informed consent to participate in the research.

\section{Consent for publication}

All patients included in this study gave a written informed consent to publish the data contained in this study.

\section{Competing interests}

The authors declare that they have no competing interests.

\section{Author details}

'Diagnostic and Interventional Radiology, Mansoura University, Mansoura, Egypt. ${ }^{2}$ Cardiothoracic Surgery, Mansoura University, Mansoura, Egypt.

Received: 21 August 2020 Accepted: 13 January 2021

Published online: 04 February 2021

\section{References}

1. Rao PS (2009) Diagnosis and management of cyanotic congenital heart disease: part I. Indian J Pediatr 76(1):57-70

2. Banderker $\mathrm{E}$, Pretorius $\mathrm{E}$, de Decker $\mathrm{R}$ (2015) The role of cardiac $C T$ angiography in the pre-and postoperative evaluation of tetralogy of Fallot SA J Radiol 9(2)

3. Lapierre C, Dubois J, Rypens F et al (2016) Tetralogy of Fallot: preoperative assessment with MR and CT imaging. Diagn Interv Imaging 97(5):531-541

4. Bu G, Miao Y, Bin J et al (2016) Comparison of 128-slice low-dose prospective ECG-gated CT scanning and trans-thoracic echocardiography for the diagnosis of complex congenital heart disease. PLoS One 11(10): e0165617

5. Silverman NH (2013) The essential echocardiographic features of tetralogy of Fallot. Cardiol Young 23(6):871-882

6. Bayraktutan U, Kantarci M, Ogul $\mathrm{H}$ et al (2013) The utility of multidetector computed tomography for evaluation of congenital heart disease. Folia Morphol (Warsz) 72(3):188-196

7. Sahu A, Slesnick TC (2017) Imaging adults with congenital heart disease part II. J Thorac Imaging 32(4):245-257

8. Wang X-M, Wu L-B, Sun C et al (2007) Clinical application of 64-slice spiral CT in the diagnosis of the tetralogy of Fallot. Eur J Radiol 64(2):296-301

9. Apostolopoulou SC, Manginas A, Kelekis NL et al (2019) Cardiovascular imaging approach in pre and postoperative tetralogy of Fallot. BMC Cardiovasc Disord 19(1):7

10. Hu B-y, Shi K, Deng Y-P et al (2017) Assessment of tetralogy of Fallotassociated congenital extracardiac vascular anomalies in pediatric patients using low-dose dual-source computed tomography. BMC Cardiovasc Disord 17(1):285

11. Barron DJ, Stickley J, Anderson RH, et al. Tetralogy of Fallot: morphological variations and implications for surgical repairt. 2019.

12. Hrusca A, Rachisan AL, Gach P et al (2016) Detection of pulmonary and coronary artery anomalies in tetralogy of Fallot using non-ECG-gated $C T$ angiography. Diagn Interv Imaging 97(5):543-548

13. Agrawal S, Soni D, Dhawan S et al (2014) Sites of right ventricular outflow tract obstruction in tetralogy of Fallot--a retrospective study. Indian Heart 43(6):455-459

14. Zakaria RH, Barsoum NR, Asaad RE et al (2011) Tetralogy of Fallot: imaging of common and uncommon associations by multidetector $C T$. Egypt $J$ Radiol Nucl Med 42(3):289-295

15. Sheikh AM, Kazmi U, Syed NH (2014) Variations of pulmonary arteries and other associated defects in tetralogy of Fallot. SpringerPlus. 3:467

16. Ramanathan R, Anumandla AK, Haramati LB et al (2014) Evaluation of the cardiac chambers on axial CT: comparison with echocardiography. J Comput Assist Tomogr 38(1):53-56

17. Chen B-B, Chen S-J, Wu M-H et al (2007) EBCT-McGoon ratio a reliable and useful method to predict pulmonary blood flow non-invasively. Chin J Radiol 32(1):1-8

18. Meinel FG, Huda W, Schoepf UJ et al (2013) Diagnostic accuracy of CT angiography in infants with tetralogy of Fallot with pulmonary atresia and major aortopulmonary collateral arteries. J Cardiovasc Comput Tomograph 7(6):367-375

19. Juneja M, Doshi D, Sahoo S et al (2014) Incidence of aortopulmonary collaterals in patients of tetralogy of Fallot with pulmonary atresia \& correlation with pulmonary artery anatomy. Indian Heart J 66:S49-S50

20. Chandrashekhar G, Sodhi KS, Saxena AK et al (2012) Correlation of 64 row MDCT, echocardiography and cardiac catheterization angiography in assessment of pulmonary arterial anatomy in children with cyanotic congenital heart disease. Eur J Radiol 81(12):4211-4217

21. Rehman R, Marhisham MC, Alwi M (2018) Stenting the complex patent ductus arteriosus in tetralogy of Fallot with pulmonary atresia: challenges and outcomes. Futur Cardiol 14(1):55-73

22. Shehata SM, Zaiton FM, Abo Warda MH et al (2017) Value of MDCT as a non- invasive modality in evaluation of pediatric congenital cardiovascular anomalies. Egypt J Radiol Nucl Med 48(2):467-478

23. Ishihara A, Funabashi N, Ozawa K et al (2016) The use of whole thoracic ECG-gated MDCT for the de novo diagnosis of isolated patent ductus arteriosus in middle aged or older subjects. Int J Cardiol 224:62-64

24. Leschka S, Oechslin E, Husmann L et al (2007) Pre-and postoperative evaluation of congenital heart disease in children and adults with 64section CT. Radiographics. 27(3):829-846

25. Beig JR, Ahmed W, Hafeez I et al (2014) Pentalogy of Fallot with a single coronary artery: a rare case report. J Tehran Heart Center 9(3):132-134

26. Goitein O, Salem Y, Jacobson J et al (2014) The role of cardiac computed tomography in infants with congenital heart disease. Isr Med Assoc J 52147(3):16

27. Verma I, Dara A, Vyas C et al (2018) Anaesthetic management of a patient with pentalogy of Fallot: a case report. Egypt J Cardiothorac Anesth 12(2): $31-34$

28. Yasunaga D, Hamon M (2015) MDCT of interatrial septum. Diagn Interv Imaging 96(9):891-899

29. Popieluszko P, Henry BM, Sanna B et al (2018) A systematic review and meta-analysis of variations in branching patterns of the adult aortic arch. J Vasc Surg 68(1):298-306.e10

30. Tawfik AM, Sobh DM, Ashamallah GA et al (2018) Prevalence and types of aortic arch variants and anomalies in congenital heart diseases. Acad Radiol

31. Hanneman K, Newman B, Chan F (2017) Congenital variants and anomalies of the aortic arch. RadioGraphics. 37(1):32-51

32. Sen S, Rao SG, Kulkarni S (2016) Rare associations of tetralogy of Fallot with anomalous left coronary artery from pulmonary artery and totally anomalous pulmonary venous connection. Cardiol Young 26(5):1017-1020

33. Chan SS, Whitehead KK, Kim TS et al (2015) Repaired tetralogy of Fallot with coexisting unrepaired partial anomalous pulmonary venous connection is associated with diminished right ventricular ejection fraction and more severe right ventricular dilation. Pediatr Radiol 45(10):1465-147

34. Türkvatan A, Tola HT, Kutlutürk N et al (2017) Low-dose computed tomographic imaging of partial anomalous pulmonary venous connection in children. World J Pediatr Congenit Heart Surg 8(5):590-596

35. Ari ME, Doğan V, Özgür S et al (2017) Persistent left superior vena cava accompanying congenital heart disease in children: experience of a tertiary care center. Echocardiography. 34(3):436-440

36. Nagasawa H, Kuwabara N, Goto H et al (2018) Incidence of persistent left superior vena cava in the normal population and in patients with congenital heart diseases detected using echocardiography. Pediatr Cardiol 39(3):484-490

37. Corno AF, FETCS F, Alahdal SA et al (2013) Systemic venous anomalies in the Middle East. Front Pediatr 1:1

38. Lim ZN, BJW C, Yong S et al (2018) Tetralogy of Fallot with right aortic arch and retro-aortic innominate vein. Front Pediatr 6(331)

\section{Publisher's Note}

Springer Nature remains neutral with regard to jurisdictional claims in published maps and institutional affiliations. 